\title{
New Twists on the Epigenetics of CpG Islands
}

\author{
Rolf Ohlsson ${ }^{1}$ and Chandrasekhar Kanduri \\ Department of Development and Genetics, Evolution Biology Centre, Uppsala University, Norbyvägen 18 A, S-752 36 Uppsala, Sweden
}

C have a significantly higher concentration of $\mathrm{CpG}$ dinucleotides than the bulk of the genome. Whereas $70 \%-80 \%$ of all $\mathrm{CpG}$ dinucleotides in the human genome are methylated, CpG islands by and large remain unmethylated (Cross and Bird 1995), with the exception of those associated with imprinted and X-linked genes (Razin and Cedar 1994). In this issue of Genome Research, Strichman-Almashanu et al. (2002) present the first successful systematic approach to generating libraries of differentially methylated and unique CpG islands and show its use in uncovering novel imprinted genes.

Methylated CpG Islands and Genomic Imprinting

Figure 1 outlines the strategy used to select fragments that were subsequently cloned and sequenced. Whereas the majority of the clones isolated in this manner corresponded to $\mathrm{CpG}$ islands, most of the several hundred clones represented CpG island repeats, such as the nontranscribed intergenic spacer of ribosomal DNA and a transposon repeat, termed SVA. The library was further pro-

'Corresponding author.

E-Mail: Rolf.OhIsson@ebc.uu.se; FAX 4618 4712683

Article and publication are at http://www.genome. org/cgi/doi/10.1101/gr.18002.

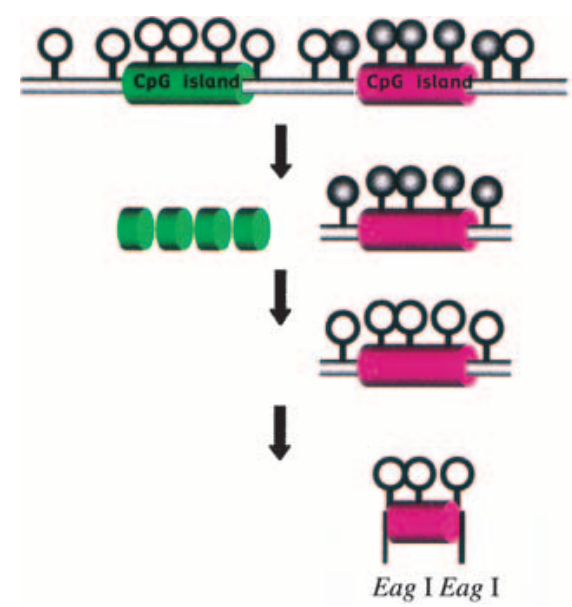

cessed, therefore, to generate clones that were all determined to be $\mathrm{CpG}$ islands. Southern analysis of the library revealed that the clones fell into two categories: those that are densely methylated on both alleles both in soma and in sperm (termed SMRs) and those differentially methylated (termed gDMRs), as determined by analyzing DNA of uniparental tissues presumably displaying epigenetic states of maternal or paternal origin.

One of the differentially methylated sequences of the CpG island library of Strichman-Almashanu et al. (2002) mapped to a previously known imprinted gene, HYMA1. Encouraged by this finding, the authors searched their library for neighboring genes that were expressed in a parent of origindependent manner. Indeed, a novel imprinted gene, termed Elongin A3, was found flanking another differentially methylated CpG island sequence of the library. This confirmed not only the supposition that differentially methylated CpG islands might be common among imprinted loci but also the feasibility of the approach of StrichmanAlmashanu et al. to identify novel imprinted genes.

\section{Genome Structure and Epigenetic Status of CpG Islands}

It has recently been proposed that $\mathrm{CpG}$ is-

DNA is restricted with Mse I and $\mathrm{Hpa}$ II.

The methylated $\mathrm{CpG}$ island (red) is relatively larger than DNA background and purifies with non-island DNA

Following subcloning, the inserts are isolated from the bacteria in an unmethylated state

Eag I digests $\mathrm{CpG}$ islands (but non-island sequences only rarely), generating $\mathrm{CpG}$ island fragments that are smaller than the background non-island DNA

Figure 1 Generation of differentially methylated CpG island library. Unfilled lollipops represent unmethylated $\mathrm{CpGs}$ and filled lollipops represent methylated CpGs. See text for details. (Revised from Fig. 1 of Strichman-Almashanu et al. 2002, with permission.) lands fall into several groups, one of which represents unique $\mathrm{CpG}$ and generally unmethylated islands associated with the $5^{\prime}$ region of housekeeping genes, whereas another includes high-copy nongene CpG islands that are dominated by Alu I repeat elements (Ponger et al. 2001). Because Alu I repeats are generally methylated and transcriptionally silent, high-copy CpG islands are predicted to be methylated. Indeed, the report of Strichman-Almashanu et al. (2002) identified one of the high-copy CpG islands (SVA) to be heavily methylated. This observation is not surprising given that repeat sequences provide signatures for de novo methylation, according to the host defense model (Bestor and Tycko 1996).

Strichman-Almashanu et al. (2002) also report the existence of a new class of unique CpG islands that are methylated on both alleles in all tissues examined. Interestingly, these CpG islands (SMRs) mapped to isochores with high GC content (>0.5), whereas the differentially methylated islands (gDMRs) were concentrated in isochores with low GC content $(<0.5)$. The class of unmethylated or differentially methylated CpG islands could stand out in a CpG-less environment and provide landmarks for various recognition events, such as the initiation of chromatin condensation by TP2 during spermiogenesis (Kundu and Rao 1996). The complexity of CpG island compartmentalization of the mammalian genome was further emphasized by the observation that the methylated highcopy CpG islands frequently localize close to telomeric ends (Strichman-Almanshanu et al. 2002), as do densely methylated nonisland CpG stretches (Brock et al. 1999), indicating some methylation-dependent role in chromosomal integrity. This deduction is supported by the observation that DNA methyltransferase, Dnmt1, is essential for genomic stability in mouse embryonic stem cells (Chen et al. 1998).

\section{Differentially Methylated CpG Islands and Expression Domains}

Perhaps the most intriguing functional aspect of the epigenetic states of CpG islands lies in their control of expression domains, as exemplified by the close apposition of differentially methylated $\mathrm{CpG}$ islands to imprinted 
genes and the distribution of unique and unmethylated $\mathrm{CpG}$ islands to the promoters of $50 \%-60 \%$ of all human genes (Cross and Bird 1995). Taken together, the data indicate that protection against de novo methylation is likely to have involved functional selection based on gene expression patterns. Whereas it is widely argued that unmethylated $\mathrm{CpG}$ islands stably propagate an open chromatin conformation that allows trans-acting factors to access pivotal promoter elements, this might be an oversimplification, as hinted at by Strichman-Almashanu et al. (2002).

Under the host-defense model, which posits that high-copy number repeats provide signals for de novo methyltransferases, methylation patterns should spread from highcopy repeats scattered throughout the genome (Bestor and Tycko 1996). The resistance of many of the unique CpG islands to waves of de novo methylation is reminiscent of how chromatin insulators are perceived to prevent silencers from accessing gene promoters. Indeed, unpublished observations (Strichman-Almanshanu et al. 2002) indicate that many CpG islands of the StrichmanAlmanshanu et al. report interact with the 11-zinc finger protein CTCF, which organizes chromatin insulator functions (Ohlsson et al. 2001). An alternative view of the role of unmethylated or differentially methylated $\mathrm{CpG}$ islands invokes, therefore, protection against silencers and/or enhancers in a gene contextdependent and stably heritable manner, as a contribution to transcriptional regulation (Fig. 2) (Hejnar et al. 2001). Interestingly, CTCF is known to participate in the generation of the methylation-free domain of the

\section{The CpG island as a methylation-dependent silencer}

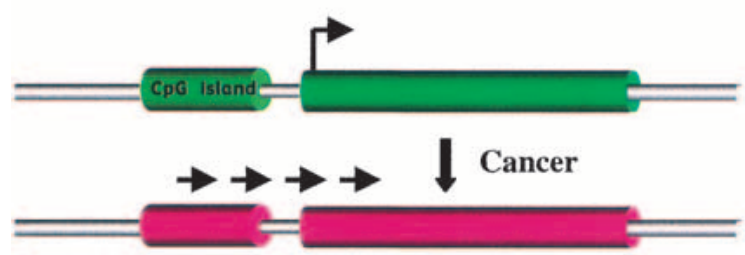

The CpG island as a methylation-dependent insulator

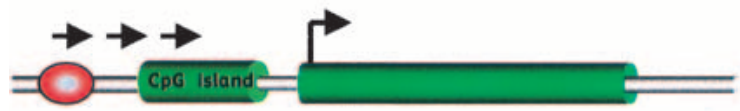

Cancer

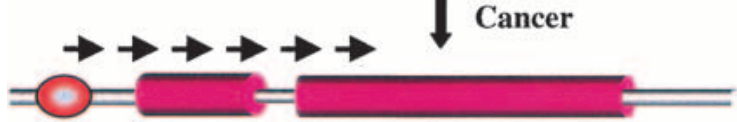

Silencer

Figure 2 De novo methylation of $\mathrm{CpG}$ islands during cancer might silence neighbouring genes in two distinct manners. maternally inherited imprinting control region flanking the $\mathrm{H} 19$ gene (V. Pant et al., unpublished observations) and, hence, might of such $\mathrm{CpG}$ islands. At the same time it should be noted that only a fraction of $\mathrm{CpG}$ array-based approach (Mukhopadhyay et with discrete subclasses of $\mathrm{CpG}$ islands. fertors interacting with $\mathrm{CpG}$-rich sequences al. 1994; Macleod et al. 1994) and other CpG-binding factors, such as hCGBP

\section{Cancer and Epigenetic States of CpG Islands}

Since the initial discovery of epigenetic lesions in cancer cells by Feinberg and Vogelstein (1983), it is now abundantly clear that cer (Issa and Baylin 1996), creating patterns of epigenetic mosaicism (Ohlsson et al. 1999). The normally unmethylated CpG islands, for example, are often methylated in to silence flanking genes during neoplasia (Fig. 2). The existence of a class of normally methylated CpG islands as reported by the complementary scenario that demethylactivation of genes. This was first shown at the MAGE-1 locus, which is normally expressed only in the male germline but is activated in human tumors (De Smet et al. 1996). However, as pointed out by Strichman-Almashanu et al. (2002), these scenarios are not straightforward because their normally methylated $\mathrm{CpG}$ islands remained methylated in cancer cells, that is, Wilms' tumors. Indeed, it has been reported that the patterns of cancer-associated methylation of $\mathrm{CpG}$ islands depend on several factors, such as cell lineage and environmental stimuli (Yan et al. 2001). The most plausible explanation for these observations posits the existence of a repertoire of factors that govern the epigenetic status of different subsets of $\mathrm{CpG}$ islands. The genetic or epigenetic inactivation of such factor functions during neoplasia might trigger epigenetic lesions, therefore, which are specific for each group of CpG islands. This in turn might expose the genes flanking such islands to silencers (or enhancers) and set the stage for various selection pathways.

\section{OUTLOOK}

Thanks to the work by Strichman-Almashanu et al. and others, a fine-scale structure of $\mathrm{CpG}$ islands is emerging, with classes of islands characterized according to genomic location and epigenetic status. However, although classifying CpG islands on the basis of epigenetic status in the germline and soma looks appealing, we must be aware that methylation status is a dynamic feature that is reshaped at several different stages during development and in cancer. The function of CpG islands during these events and the contribution of their epigenetic states to the structure and function of the genome will be sure to provide fresh insight into the mechanisms of development and disease.

\section{REFERENCES}

Bestor, T. and Tycko, B. 1996. Nat. Genet. 12: 363-367.

Brandeis, M., Frank, D., Keshet, I., Siegfried, Z., Mendelsohn, M., Nemes, A., Temper, V., Razin, A., and Cedar, H. 1994. Nature 371: 435-438.

Brock, G., Charlton, J., and Bird, A. 1999. Gene 240: 269-277.

Chen, R., Pettersson, U., Beard, C., Jackson-Grusby, L., and Jaenisch, R. 1998. Nature 395: 89-93.

Cross, S. and Bird, A. 1995. Curr. Opin. Genet. Dev. 5: $309-314$.

De Smet, C., De Backer, O., Faraoni, I., Lurquin, C., Brasseur, F., and Boon, T. 1996. Proc. Natl. Acad. Sci. 93: 7149-7153.

Feinberg, A. and Vogelstein, B. 1983. Nature 301: 89-92.

Hejnar, J., Hajkova, P., Plachy, J., Elleder, D. Stepanets, V., and Svoboda, J. 2001. Proc. Natl. Acad. Sci. USA 98: 565-569.

Issa, J.-P. J. and Baylin, S. B. 1996. Nat. Med. 2: 281-282.

Kundu, T. and Rao, M. 1996. Biochemistry 35: 15626-15632.

Macleod, D., Charlton, J., Mullins, J., and Bird, A.P. 1994. Genes Dev. 8: 2282-2292.

Ohlsson, R., Cui, H., He, L., Pfeifer, S., Jiang, S., Feinberg, A. P., and Hedborg, F. 1999. Cancer Res. 59: 3889-3892.

Ohlsson, R., Renkawitz, R., and Lobanenkov, V. 2001. Trends Genet. 17: 520-527.

Ponger, L., Duret, L., and Mouchiroud, D. 2001. Genome Res. 11: 1854-1860.

Razin, A. and Cedar, H. 1994. Cell 77: 473-476.

Strichman-Almanshanu, L., Lee, R., Onyango, P., Perlman, E., Flam, F., Frieman, M., and Feinberg, A. 2002. Genome Res. X, Y.

Voo, K.S,. Carlone, D.L., Jacobsen, B.M., Flodin, A., and Skalnik, D.G. 2000. Mol. Cell. Biol. 20 2108-2121

Yan, P., Chen, C., Shi, H., Rahmatpanah, F., Wei, S., Caldwell, C., and Huang, T. 2001. Cancer Res. 61: 8375-8380. 


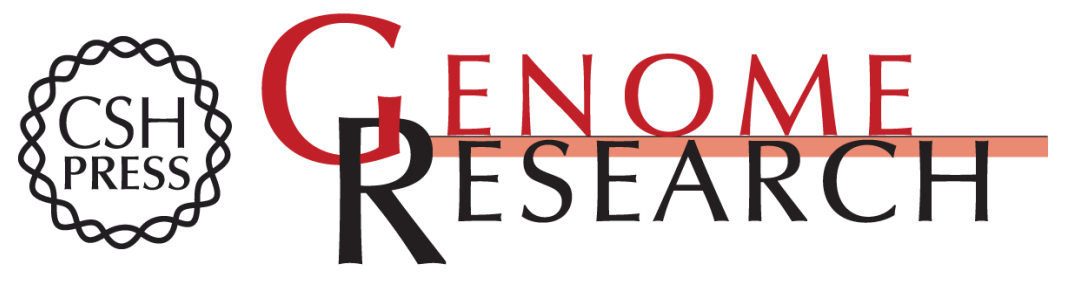

\section{New Twists on the Epigenetics of CpG Islands}

Rolf Ohlsson and Chandrasekhar Kanduri

Genome Res. 2002 12: 525-526

Access the most recent version at doi:10.1101/gr.18002

References This article cites 17 articles, 7 of which can be accessed free at:

http://genome.cshlp.org/content/12/4/525.full.html\#ref-list-1

\section{License}

Email Alerting Receive free email alerts when new articles cite this article - sign up in the box at the Service top right corner of the article or click here.

\section{Affordable, Accurate Sequencing.}

To subscribe to Genome Research go to: https://genome.cshlp.org/subscriptions 